\title{
Preface
}

\section{Georg Pflug}

The 7th Conference on Computational Management Science was held at the University of Vienna, July 28th-30th, 2010. The previous CMS conferences were in Neuchatel (2004), Gainesville (2005), Amsterdam (2006), Geneva (2007), London (2008) and again Geneva (2009).

Five plenary and 120 invited or contributed talks were given at the conference. In this volume, selected papers are collected. They cover the typical scope of Computational Management Science, from modeling over algorithms to Computer implementations.

Out of the plenary presenters, Claudia Sagastizabal and Mikhail Solodov show how the bundle method can be applied to two stage stochastic programs with recourse, called the generation expansion problem. Existing power plants may be extended and/or new power plants may be built under constraints for air and water pollution and a minimal cost objective. The uncertainty not revealed at the planning time concerns demand and air pollution limits.

The second plenary paper is a survey of multi-parametric programming and control algorithms by Pistikopoulos, Dominguez, Panos, Kouramas and Chinchuluun. It is demonstrated, how the general multi-parametric model specializes for nonlinear programming, mixed integer programming, bi- and multi-level programming, dynamic programming and multiobjective programming. The approaches are illustrated by various applications in different fields.

The invited paper authored by Nagurney, Masoumi and Min Yu deals with supply chain optimization, in particular for human blood, which is an urgent and perishable good. The model is formulated as a two-criteria problem, the criteria being costs and supply risk.

\footnotetext{
G. Pflug (ه)

Vienna, Austria

e-mail: georg.pflug@univie.ac.at
} 
Krawczyk and Sissons compare the two basic paradigms of decision making: optimal decisions versus satisfactory decisions. As an example, they consider the problem of determining the price for tickets for a sport event, where not only the profit, but also some sales target has to be considered.

Herminia Calvete's paper, jointly written with del Pozo and Iranzo is concerned with a network optimization problem, where one aims at finding the quickest path under constraints for capacity. Moreover, the reliability of the path is often an issue (for instance in telecommunication networks) and therefore reliability can be added as an additional constraint.

Le Hoai Minh, Yassine and Moussi consider the problem of optimal transportation in a container port, which turns out to be a large mixed integer problem. The authors demonstrate how the problem can be rewritten in penalty form and then reformulated as a difference of convex function problem, for which iterative algorithms exist. Numerical experiments show the quality of the approach.

The last paper deals with implementations of linear optimization, which is the most important problem in the papers mentioned above. Eleyat and Natvig consider interior point linear programming solver algorithms implemented on a heterogeneous processor and show how a sparse Cholesky decomposition works on a parallel architecture.

As guest editor of this volume, I want to thank all referees for the work they have done and the editor-in-chief Professor Rustem for his support and patience.

Georg Pflug

Vienna, December 2011 